\title{
RELATIVE CONGRUENCE FORMULAS AND DECOMPOSITIONS IN QUASIVARIETIES
}

\author{
MIGUEL A. CAMPERCHOLI AND JAMES G. RAFTERY
}

\begin{abstract}
Quasivarietal analogues of uniform congruence schemes are discussed, and their relationship with the equational definability of principal relative congruences (EDPRC) is established, along with their significance for relative congruences on subalgebras of products. Generalizing the situation in varieties, we prove that a quasivariety is relatively ideal iff it has EDPRC; it is relatively filtral iff it is relatively semisimple with EDPRC. As an application, it is shown that a finitary sentential logic, algebraized by a quasivariety $\mathrm{K}$, has a classical inconsistency lemma if and only if $\mathrm{K}$ is relatively filtral and the subalgebras of its nontrivial members are nontrivial. A concrete instance of this result is exhibited, in which $\mathrm{K}$ is not a variety. Finally, for quasivarieties $\mathrm{M} \subseteq \mathrm{K}$, we supply some conditions under which $M$ is the restriction to $K$ of a variety, assuming that $\mathrm{K}$ has EDPRC.
\end{abstract}

\section{INTRODUCTION}

This paper concerns quasivarieties of algebras, but it arose from questions about algebraizable (sentential) logics, in the sense of Blok and Pigozzi [8]. The logical motivation is explained briefly below, although no subsequent argument depends on it.

As in [8], logics $\vdash$ are assumed finitary, i.e., whenever $\Gamma \vdash \alpha$, then $\Gamma^{\prime} \vdash \alpha$ for some finite $\Gamma^{\prime} \subseteq \Gamma$. A set $\Gamma$ of formulas is said to be inconsistent in $\vdash$ if $\Gamma \vdash \alpha$ for every formula $\alpha$. Abstracting a familiar feature of classical propositional logic (CPL), we say that $\vdash$ has a classical inconsistency lemma if, for each positive integer $n$, there exists a set $\Psi_{n}$ of formulas in the variables $x_{1}, \ldots, x_{n}$, such that, for any set $\Gamma \cup\left\{\alpha_{1}, \ldots, \alpha_{n}\right\}$ of formulas,

(1) $\Gamma \cup\left\{\alpha_{1}, \ldots, \alpha_{n}\right\}$ is inconsistent in $\vdash$ iff $\Gamma \vdash \Psi_{n}\left(\alpha_{1}, \ldots, \alpha_{n}\right)$;

(2) $\Gamma \cup \Psi_{n}\left(\alpha_{1}, \ldots, \alpha_{n}\right)$ is inconsistent in $\vdash$ iff $\Gamma \vdash\left\{\alpha_{1}, \ldots, \alpha_{n}\right\}$.

The realizations of (1) and (2) in CPL are

$$
\begin{aligned}
& \Gamma \cup\left\{\alpha_{1}, \ldots, \alpha_{n}\right\} \text { is inconsistent iff } \Gamma \vdash \neg\left(\alpha_{1} \wedge \cdots \wedge \alpha_{n}\right) ; \\
& \Gamma \cup\left\{\neg\left(\alpha_{1} \wedge \cdots \wedge \alpha_{n}\right)\right\} \text { is inconsistent iff } \Gamma \vdash\left\{\alpha_{1}, \ldots, \alpha_{n}\right\} .
\end{aligned}
$$

2010 Mathematics Subject Classification. Primary: 08C15; Secondary: 03G27.

Key words and phrases. Quasivariety, filtral, semisimple, EDPC, ideal, inconsistency lemma.

The second author was supported in part by the National Research Foundation of South Africa (UID 85407). 
The case $n=1$ is of course sufficient for $\mathbf{C P L}$, but the remaining cases are not generally redundant (see [52]).

Familiar logics are often algebraized by varieties, but in many cases, the algebraic counterpart is merely a quasivariety, and these classes will constitute our framework. The following 'bridge theorem' was established recently.

Theorem 1.1. ([52]) Let $\mathrm{K}$ be a quasivariety that algebraizes a logic $\vdash$. Then $\vdash$ has a classical inconsistency lemma iff $\mathrm{K}$ is relatively semisimple, with equationally definable principal relative congruences (EDPRC), and for every $\boldsymbol{A} \in \mathrm{K}$, the total congruence $A^{2}:=A \times A$ of $\boldsymbol{A}$ is compact in the lattice of all K-congruences of $\boldsymbol{A}$.

There are quasivarieties, other than varieties, that meet all the demands in Theorem 1.1, and some of them algebraize logics to which attention has been drawn in the literature; see Example 6.7 below.

Now it has long been known that a variety is semisimple with EDPC iff it is filtral $[27,28]$, and the notion of filtrality extends naturally to quasivarieties (Definitions 4.2 and 6.1). One might therefore hope to reformulate Theorem 1.1 as a connection between classical inconsistency lemmas and relatively filtral quasivarieties - based on an expected equivalence

$$
\text { relatively filtral }=\text { relatively semisimple with EDPRC, }
$$

which specializes, in varieties, to the aforementioned

$$
\text { filtral }=\text { semisimple with EDPC. }
$$

It seems, however, that there is no literature on relatively filtral (and more generally, relatively ideal) quasivarieties - perhaps because of definitional conflicts in the varietal tradition and the limited visibility of sources that resolve them. Another possible factor is that (4) emerges gradually in [27] from Maltsev's familiar analysis of absolute congruence generation, which motivates the 'uniform congruence schemes' of Fried, Grätzer and Quackenbush, but which does not apply to the relative congruences determined by quasivarieties.

The idea of uniform congruence schemes can be adapted to quasivarieties, via descriptions of relative congruence generation. We connect the resulting uniform relative congruence formulas with EDPRC in Section 3, and with the behaviour of subalgebras of products in Section 5, after widening the scope of some results of Kiss [36] in Section 4. The equivalence in (3) is deduced in Theorem 6.3. Also, the compactness demand appearing in Theorem 1.1 can be made more transparent [14], so we obtain the following improved characterization, on purely algebraic grounds.

Theorem 1.2. Let $\mathrm{K}$ be a quasivariety that algebraizes a logic $\vdash$. Then $\vdash$ has a classical inconsistency lemma iff $\mathrm{K}$ is relatively filtral and the subalgebras of its nontrivial members are nontrivial. 
Finally, we partially address the question: in a quasivariety $\mathrm{K}$ with ED$\mathrm{PRC}$, when must a subquasivariety be the restriction to $\mathrm{K}$ of some variety? (See Theorems 5.6 and 6.6 and Corollary 5.7.)

\section{Quasivarieties}

Recall that a quasivariety is the model class of some set of pure quasiequations $\left(\&_{i<n} \gamma_{i} \approx \delta_{i}\right) \Longrightarrow \gamma \approx \delta$ in an algebraic signature. Here $n \in \omega$, i.e., quasi-equations have finite length and encompass equations. The class operator symbols $\mathbb{I}, \mathbb{H}, \mathbb{S}, \mathbb{P}, \mathbb{P}_{\mathbb{S}}, \mathbb{P}_{\mathbb{R}}$ and $\mathbb{P}_{\mathbb{U}}$ stand for closure under isomorphic and homomorphic images, subalgebras, direct, subdirect and reduced products, and ultraproducts, respectively. A class $\mathrm{M}$ of similar algebras is a quasivariety iff it is closed under $\mathbb{I}, \mathbb{S}$ and $\mathbb{P}_{\mathbb{R}}$ (equivalently, under $\mathbb{I}, \mathbb{S}, \mathbb{P}$ and $\mathbb{P}_{\mathbb{U}}$ ); the smallest such class containing $M$ is $\mathbb{I S P}_{\mathbb{R}}(\mathrm{M})=\mathbb{I S P P}_{\mathbb{U}}(\mathrm{M})=$ $\mathbb{I P}_{\mathbb{S}} \mathbb{S P}_{\mathbb{U}}(\mathrm{M})[45,32] . \mathrm{M}$ is a universal class (i.e., the model class of some set of universal first order sentences) iff it is closed under $\mathbb{I}, \mathbb{S}$ and $\mathbb{P}_{\mathbb{U}}$. Moreover, $\mathbb{P}_{\mathbb{U}}(\mathrm{M}) \subseteq \mathbb{I}(\mathrm{M})$ if $\mathrm{M}$ is a finite set of finite algebras. All these facts can be found in any of $[13,30,31]$.

For the remainder of this paper, $\mathrm{K}$ denotes a given quasivariety of algebras. Thus, $\mathbb{H}(\mathrm{K})$ is the smallest variety containing $\mathrm{K}$. All algebras considered are assumed to have K's type, but they need not be members of $\mathrm{K}$.

The K-congruences (a.k.a. relative congruences) of an algebra $\boldsymbol{A}$ are the congruences $\theta$ such that $\boldsymbol{A} / \theta \in \mathrm{K}$. They form an algebraic lattice $\boldsymbol{C o n}_{\mathrm{K}} \boldsymbol{A}$, ordered by inclusion, in which meets are intersections and the compact elements are just the finitely generated $\mathrm{K}$-congruences. We denote by $\Theta_{\mathrm{K}}^{\boldsymbol{A}} X$ the least K-congruence of $\boldsymbol{A}$ containing a subset $X$ of $A^{2}$. When $X$ is a singleton $\{\langle a, b\rangle\}$, we write $\Theta_{\mathrm{K}}^{\boldsymbol{A}}(a, b)$ for the principal $\mathrm{K}$-congruence $\Theta_{\mathrm{K}}^{\boldsymbol{A}} X$. Joins in $\boldsymbol{C o n}_{\mathrm{K}} \boldsymbol{A}$ may exceed those taken in the lattice $\boldsymbol{C o n} \boldsymbol{A}$ of (absolute) congruences of $\boldsymbol{A}$. (In fact, $\boldsymbol{C o n}_{\mathrm{K}} \boldsymbol{A}$ is a sublattice of $\boldsymbol{C o n} \boldsymbol{A}$ for all $\boldsymbol{A} \in \mathrm{K}$ iff $\mathrm{K}$ is a variety [15].) When $\mathrm{K}$ is a variety and $\boldsymbol{A} \in \mathrm{K}$, the congruences and K-congruences of $\boldsymbol{A}$ coincide, so the prefixes and subscripts can be dropped. From this point of view, relative congruences generalize absolute ones.

The result below is due to Campercholi and Vaggione [14]; it was proved first for varieties by Kollár [40].

Theorem 2.1. ([14]) The following conditions on $\mathrm{K}$ are equivalent.

(i) For every $\boldsymbol{A} \in \mathrm{K}$, the total relation $A^{2}$ is compact in $\boldsymbol{C o n}_{\mathrm{K}} \boldsymbol{A}$.

(ii) All subalgebras of nontrivial members of $\mathrm{K}$ are nontrivial.

The quasivariety $\mathrm{K}$ is said to be relatively congruence distributive (RCD) if its members have distributive lattices of K-congruences. It has the relative congruence extension property $(\mathrm{RCEP})$ if every $\mathrm{K}$-congruence on a subalgebra $\boldsymbol{A}$ of an algebra $\boldsymbol{B} \in \mathrm{K}$ is the restriction $\left.\theta\right|_{\boldsymbol{A}}:=A^{2} \cap \theta$ of some $\theta \in \operatorname{Con}_{\mathrm{K}} \boldsymbol{B}$. It was shown in [9] that $\mathrm{K}$ has the RCEP iff, whenever $\boldsymbol{B} \in \mathrm{K}$ 
and $\boldsymbol{A} \in \mathbb{S}(\boldsymbol{B})$, with $a, b \in A$, then $\Theta_{\mathrm{K}}^{\boldsymbol{A}}(a, b)=\left.\Theta_{\mathrm{K}}^{\boldsymbol{B}}(a, b)\right|_{\boldsymbol{A}}$. (For varieties, this was proved by Day [21].)

An algebra $\boldsymbol{A} \in \mathrm{K}$ is said to be $\mathrm{K}$-subdirectly irreducible, or finitely $\mathrm{K}$ subdirectly irreducible if, in $\boldsymbol{C o n}_{\mathrm{K}} \boldsymbol{A}$, the relation $\operatorname{id}_{A}:=\{\langle a, a\rangle: a \in A\}$ is completely meet-irreducible, or (finitely) meet-irreducible, respectively. Recall that $\boldsymbol{A}$ is finitely subdirectly irreducible (in the absolute sense) iff id ${ }_{A}$ is meet-irreducible in $\boldsymbol{C o n} \boldsymbol{A}$. We denote by $\mathrm{K}_{R S I}, \mathrm{~K}_{R F S I}$ and $\mathrm{K}_{F S I}$ the respective classes of $\mathrm{K}$-subdirectly irreducible, finitely $\mathrm{K}$-subdirectly irreducible, and finitely subdirectly irreducible members of $\mathrm{K}$. Thus, $\mathrm{K}_{R S I} \cup \mathrm{K}_{F S I} \subseteq$ $\mathrm{K}_{R F S I}$, and $\mathrm{K}_{R S I}$ contains no trivial algebra. The following variant of the Birkhoff subdirect decomposition theorem holds.

Theorem 2.2. ([51, Theorem 1.1]) $\mathrm{K}=\mathbb{I P}_{\mathbb{S}}\left(\mathrm{K}_{R S I}\right)$.

Theorem 2.3. Let $\mathrm{K}$ be the smallest quasivariety containing a class $\mathrm{X}$.

(i) ([19]) Every nontrivial member of $\mathrm{K}_{R F S I}$ belongs to $\mathbb{I S P}_{\mathbb{U}}(\mathrm{X})$.

If $\mathrm{K}$ is relatively congruence distributive, then

(ii) ([22]) $\mathrm{K}_{R F S I}=\mathrm{K}_{F S I}$, and

(iii) ([19]) $\mathrm{K}_{R F S I}$ is a universal class iff, for all $\boldsymbol{A} \in \mathrm{K}$, the intersection of any two compact $\mathrm{K}$-congruences of $\boldsymbol{A}$ is compact.

An algebra $\boldsymbol{A} \in \mathrm{K}$ is said to be $\mathrm{K}$-simple if it has just two K-congruences (whence it is $\mathrm{K}$-subdirectly irreducible). If every $\mathrm{K}$-subdirectly irreducible member of $\mathrm{K}$ is $\mathrm{K}$-simple, then $\mathrm{K}$ is said to be relatively semisimple.

A classical example of a relatively semisimple RCD quasivariety is the class CSR of unital commutative rings having no nonzero nilpotent element. (The $\mathbf{S}$ stands for semiprime.) This is not a variety, as it includes $\mathbb{Z}$ but not $\mathbb{Z}_{4}$. Distributivity follows from results in [54] (or from stronger ones in $[34,35])$. Theorem 2.3 is witnessed by the fact that $\mathrm{CSR}_{R F S I}$ and $\mathrm{CSR}_{F S I}$ are both the (universal) class of all integral domains, while $\operatorname{CSR}_{R S I}$ is the class of all fields, whence CSR is relatively semisimple.

\section{UNIFORM RELATIVE CONGRUENCE FORMULAS AND EDPRC}

We use $\boldsymbol{T} \boldsymbol{m}$ to denote an absolutely free algebra generated by an infinite set of variables. The generation of K-congruences can be characterized as follows. (The criterion below is simpler than [30, Proposition 1.4.16] and $[19$, Lemma 2.2], as it involves only one quasi-equation and does not require $\boldsymbol{A} \in \mathrm{K}$.)

Lemma 3.1. ([11, Lemma 4.2]) For any algebra $\boldsymbol{A}$, with $X \cup\{\langle c, d\rangle\} \subseteq A^{2}$, we have $\langle c, d\rangle \in \Theta_{\mathrm{K}}^{\boldsymbol{A}} X$ iff there exist a quasi-equation

$$
\left(\&_{i<n} \gamma_{i} \approx \delta_{i}\right) \Longrightarrow \gamma \approx \delta,
$$

satisfied by $\mathrm{K}$, and a homomorphism $h: \boldsymbol{T} \boldsymbol{m} \rightarrow \boldsymbol{A}$ such that $h(\gamma)=c$ and $h(\delta)=d$ and, for all $i<n,\left\langle h\left(\gamma_{i}\right), h\left(\delta_{i}\right)\right\rangle \in X$ or $h\left(\gamma_{i}\right)=h\left(\delta_{i}\right)$. 
Corollary 3.2. If $g: \boldsymbol{A} \rightarrow \boldsymbol{B}$ is a homomorphism, and $\langle c, d\rangle \in \Theta_{\mathrm{K}}^{\boldsymbol{A}} X$, then $\langle g(c), g(d)\rangle \in \Theta_{\mathrm{K}}^{B}\left\{\left\langle g(x), g\left(x^{\prime}\right)\right\rangle:\left\langle x, x^{\prime}\right\rangle \in X\right\}$.

Proof. Use the homomorphism $g \circ h$, where $h$ is as in Lemma 3.1.

The generation of principal (absolute) congruences is described by Maltsev's Lemma [44]; cf. [13, Lemma V.3.1]. For principal relative congruences, a different specialization of Lemma 3.1 is needed. The letters $u, v, w, x, y, z$ (with or without indices) shall be used to denote distinct variables.

Recall that a primitive positive (or $p p$ ) formula (in the language of $\mathrm{K}$ ) is a first order formula $\varphi$ of the form $\exists u_{1} \ldots \exists u_{m} \psi$, where $\psi$ is a conjunction of equations. Thus, a quantifier-free pp formula is the same thing as a (finite) conjunction of equations. As usual, the notation $\varphi\left(x_{1}, \ldots, x_{n}\right)$ signifies that the free variables of $\varphi$ are among $x_{1}, \ldots, x_{n}$. We make standard use of the satisfaction symbol $\mid=$ and substitution conventions.

Definition 3.3. A pp formula $\varphi(x, y, z, w)$ is called a principal $\mathrm{K}$-congruence formula if

$$
\mathrm{K} \models \varphi(x, x, z, w) \Longrightarrow z \approx w .
$$

Fixing the sequence $x, y, z, w$ of potential free variables, we denote the set of all principal $\mathrm{K}$-congruence formulas $\varphi(x, y, z, w)$ by $\Phi_{\mathrm{K}}(x, y, z, w)$, or by $\Phi_{\mathrm{K}}$ when no confusion is likely.

The terminology is justified by the next lemma, which has almost the same content as [30, Proposition 1.4.15] and [20, Theorem 2.1]. (The demand $\boldsymbol{A} \in \mathrm{K}$ was imposed in those sources, and earlier in [29, p. 206], but it can be dropped, as the result follows easily from Lemma 3.1. When $\mathrm{K}$ is a variety and $\boldsymbol{A} \in \mathrm{K}$, the statement of Lemma 3.4 simplifies that of [53, Proposition 0.3].)

Lemma 3.4. For any algebra $\boldsymbol{A}$, with $a, b, c, d \in A$, the following conditions are equivalent:

(i) $\langle c, d\rangle \in \Theta_{\mathrm{K}}^{\boldsymbol{A}}(a, b)$;

(ii) there exists $\varphi \in \Phi_{\mathrm{K}}(x, y, z, w)$ such that $\boldsymbol{A} \models \varphi(a, b, c, d)$.

It may happen that all instances of principal K-congruence generation in $\mathrm{K}$ are witnessed, in this sense, by the same formula $\varphi$. That thought leads to quasivarietal analogues of the 'uniform congruence schemes' of Fried, Grätzer and Quackenbush [27].

Definition 3.5. A uniform relative congruence formula (URCF) for $\mathrm{K}$ is a formula $\varphi \in \Phi_{\mathrm{K}}(x, y, z, w)$ such that for each $\boldsymbol{A} \in \mathrm{K}$ and $a, b, c, d \in A$,

$$
\text { if }\langle c, d\rangle \in \Theta_{\mathrm{K}}^{\boldsymbol{A}}(a, b) \text {, then } \boldsymbol{A}=\varphi(a, b, c, d) \text {. }
$$

(The converse follows from Lemma 3.4.) We then say that $\varphi$ defines principal relative congruences in $\mathrm{K}$. 
We call $\varphi$ a restricted URCF for $\mathrm{K}$ if, in addition, it is quantifier-free.

If $\mathrm{K}$ is a variety, we drop the word 'relative' and use the acronym UCF.

For example, the variety BA of Boolean algebras has a restricted UCF, viz.

$$
\varphi(x, y, z, w):=(x \leftrightarrow y) \rightarrow(z \leftrightarrow w) \approx \top .
$$

Indeed, $\mathrm{BA} \models u \leq v \Longleftrightarrow u \rightarrow v \approx \top$, while for any $\boldsymbol{A} \in \mathrm{BA}$, we have

$$
\langle c, d\rangle \in \Theta^{\boldsymbol{A}}(a, b) \text { iff } a \leftrightarrow b \leq c \leftrightarrow d,
$$

and if $a \leftrightarrow a \leq c \leftrightarrow d$, then $c=d$. The variety CR of unital commutative rings has a $\mathrm{UCF}$

$$
\varphi(x, y, z, w):=\exists v((x-y) v \approx z-w)
$$

because, for each $\boldsymbol{R} \in \mathrm{CR}$, we have $\langle c, d\rangle \in \Theta^{\boldsymbol{R}}(a, b)$ iff $(a-b) r=c-d$ for some $r \in R$, while if $(a-a) r=c-d$ for some $r \in R$, then $c=d$ (cf. [2]). But CR lacks a restricted UCF, by Theorems 3.9 and 3.8 below, as it is not congruence distributive (nor congruence extensible).

Corollary 3.6. Suppose $\mathrm{K}$ has the $R C E P$, and let $\boldsymbol{A} \in \mathrm{K}$, with $a, b, c, d \in A$. Then $\langle c, d\rangle \in \Theta_{\mathrm{K}}^{\boldsymbol{A}}(a, b)$ iff there is a quantifier-free formula $\varphi \in \Phi_{\mathrm{K}}(x, y, z, w)$ such that $\boldsymbol{A}=\varphi(a, b, c, d)$. (Note that $\varphi$ is a conjunction of equations.)

Consequently, if a quasivariety with the RCEP has a URCF, then it has a restricted $U R C F$.

Proof. In the first assertion, sufficiency follows from Lemma 3.4. Conversely, suppose $\langle c, d\rangle \in \Theta_{\mathrm{K}}^{\boldsymbol{A}}(a, b)$ and let $\boldsymbol{S}$ be the subalgebra of $\boldsymbol{A}$ generated by $\{a, b, c, d\}$. By the RCEP, $\langle c, d\rangle \in \Theta_{\mathrm{K}}^{S}(a, b)$, so by Lemma 3.4, there exists

$$
\varphi^{\prime}:=\exists u_{1} \ldots \exists u_{n} \beta(x, y, z, w, \vec{u}) \in \Phi_{\mathrm{K}}(x, y, z, w)
$$

such that $\beta$ is quantifier-free and $\boldsymbol{S}=\varphi^{\prime}(a, b, c, d)$. Every element of $\boldsymbol{S}$ has the form $\gamma^{\boldsymbol{A}}(a, b, c, d)$ for some term $\gamma$, so there are terms $\gamma_{1}, \ldots, \gamma_{n}$ in $x, y, z, w$ such that $\boldsymbol{S} \models \beta\left(a, b, c, d, \gamma_{1}(a, b, c, d), \ldots, \gamma_{n}(a, b, c, d)\right)$.

Then $\varphi:=\beta\left(x, y, z, w, \gamma_{1}(x, y, z, w), \ldots, \gamma_{n}(x, y, z, w)\right) \in \Phi_{\mathrm{K}}(x, y, z, w)$ has the desired property. As $\varphi$ is a conjunction of equations, the second assertion of the corollary follows.

In the sequel, $\mathrm{EDP}[\mathrm{R}] \mathrm{C}$ stands for equationally definable principal [relative] congruences.

Definition 3.7. We say that $\mathrm{K}$ has parameterized $E D P R C$ if there exists a set $\Sigma(x, y, z, w)$ of pp formulas in the free variables $x, y, z, w$ such that, for each $\boldsymbol{A} \in \mathrm{K}$ and $a, b, c, d \in A$, we have $\langle c, d\rangle \in \Theta_{\mathrm{K}}^{\boldsymbol{A}}(a, b)$ iff $\boldsymbol{A}=\Sigma(a, b, c, d)$. We say that $\mathrm{K}$ has $E D P R C$ if, moreover, the formulas in $\Sigma$ are quantifier-free (i.e., conjunctions of equations). Note that $\Sigma$ is not assumed finite. 
Again, we drop the ' $\mathrm{R}$ ' in 'EDPRC' when $\mathrm{K}$ is known to be a variety. Natural examples of quasivarieties with EDPRC that are not varieties can be found in $[20,55]$. Another one will be discussed in Example 6.7.

Theorem 3.8. ([10]) Every quasivariety with EDPRC is relatively congruence distributive and has the RCEP.

The notions in Definition 3.7 were developed first for varieties [27]. They were extended to quasivarieties in the late 1980s, but uniform congruence schemes were not (although [18, Chapter Q] generalizes some results from [27]). Clearly, if $\mathrm{K}$ has a URCF $\varphi$, then it has parameterized EDPRC (with $\Sigma=\{\varphi\}$ ); the same applies to a restricted URCF and EDPRC. The converses are not obvious, even when $\Sigma$ is finite, as (5) is not immediate for $\varphi \in \Sigma$. We show, however, that the converses are true, and that $\Sigma$ can always be chosen finite. These outcomes have varietal analogues in [27].

Theorem 3.9. A quasivariety has a URCF iff it has parameterized EDPRC. It has a restricted URCF iff it has EDPRC.

Proof. Suppose $\Sigma$ witnesses parameterized EDPRC for K. Then Lemma 3.4 yields $\mathrm{K}=\Sigma(x, y, z, w) \Longrightarrow \bigvee \Phi_{\mathrm{K}}(x, y, z, w)$, by which we mean that for each $\boldsymbol{A} \in \mathrm{K}$ and $a, b, c, d \in A$, if $\boldsymbol{A}=\Sigma(a, b, c, d)$, then there exists $\varphi \in \Phi_{\mathrm{K}}$ such that $\boldsymbol{A} \models \varphi(a, b, c, d)$. Consequently, by the Compactness Theorem of first order logic, $\mathrm{K}=\sigma(x, y, z, w) \Longrightarrow \varphi^{\prime}(x, y, z, w)$ for some finite conjunction $\sigma$ of formulas in $\Sigma$ and some finite disjunction $\varphi^{\prime}$ of formulas in $\Phi_{\mathrm{K}}$. Thus, $\mathrm{K}=\sigma(x, x, y, z) \Longrightarrow z \approx w$, by definition of $\Phi_{\mathrm{K}}$. Now $\sigma$ is logically equivalent to a pp formula, which is therefore a URCF for $\mathrm{K}$.

The second assertion follows by the same argument, but using Corollary 3.6 (and Theorem 3.8) instead of Lemma 3.4.

From the proof of Theorem 3.9 we obtain an immediate corollary:

Corollary 3.10. If $\Sigma$ witnesses [parameterized] EDPRC for the quasivariety $\mathrm{K}$, then so does some finite subset of $\Sigma$.

The binary join operation of $\boldsymbol{C o n}_{\mathrm{K}} \boldsymbol{A}$ is denoted by $+_{\mathrm{K}}^{\boldsymbol{A}}$ in the next lemma.

Lemma 3.11. ([10,19]) Let $\boldsymbol{A}$ be an algebra, with $\theta \in C_{\mathrm{K}} \boldsymbol{A}$ and $a, b \in A$. Then $\left(\theta+{ }_{\mathrm{K}}^{\boldsymbol{A}} \Theta_{\mathrm{K}}^{\boldsymbol{A}}(a, b)\right) / \theta=\Theta_{\mathrm{K}}^{\boldsymbol{A} / \theta}(a / \theta, b / \theta)$.

Theorem 3.12. Let $\mathrm{K}$ have parameterized EDPRC. Then, for each integer $n>0$, there is a pp formula $\sigma_{n}$ in $2 n+2$ free variables such that, whenever $\boldsymbol{A} \in \mathrm{K}$ and $\vec{a}=a_{1}, \ldots, a_{n} \in A$ and $\vec{b}=b_{1}, \ldots, b_{n} \in A$ and $c, d \in A$, then

$$
\langle c, d\rangle \in \Theta_{\mathrm{K}}^{\boldsymbol{A}}\left\{\left\langle a_{1}, b_{1}\right\rangle, \ldots,\left\langle a_{n}, b_{n}\right\rangle\right\} \text { iff } \boldsymbol{A}=\sigma_{n}(\vec{a}, \vec{b}, c, d) .
$$

If $\mathrm{K}$ has EDPRC, then $\sigma_{n}$ can be chosen to be a conjunction of equations. 
Proof. Proceed by induction on $n$. The base case follows from the definition of [parameterized] EDPRC and Corollary 3.10. At the inductive step, use Lemma 3.11, with $\theta=\Theta_{\mathrm{K}}^{\boldsymbol{A}}\left\{\left\langle a_{i}, b_{i}\right\rangle: i=1, \ldots, n-1\right\}$ and $\langle a, b\rangle=\left\langle a_{n}, b_{n}\right\rangle$.

A note about terminology is appropriate here. A variety has a '[restricted] uniform congruence scheme' in the sense of [27] iff it has a [restricted] UCF in the sense of Definition 3.5. This follows immediately from Theorem 3.9 and $[27$, Theorems 3.3, 4.5]. The reader is warned that, in [27], the ' $R$ ' in the acronym 'URCS' stood for 'restricted', not 'relative'. Also, 'parameterized EDPC' and 'EDPC' were called 'EDPC' and 'restricted EDPC (REDPC)', respectively. Definition 3.7 has since become standard, except that $\Sigma$ is sometimes assumed finite - inessentially, in view of Corollary 3.10.

\section{Product, ideal and filtral Relative Congruences}

We turn now to the relationship between EDPRC and the behaviour of relative congruences on subalgebras of direct products. Given a homomorphism $h: \boldsymbol{A} \rightarrow \boldsymbol{B}$, and $\theta \in \operatorname{Con} \boldsymbol{B}$, recall that

$$
h^{-1}[\theta]:=\left\{\left\langle a, a^{\prime}\right\rangle \in A^{2}:\left\langle h(a), h\left(a^{\prime}\right)\right\rangle \in \theta\right\} \in \operatorname{Con} \boldsymbol{A},
$$

and if $\theta \in \operatorname{Con}_{\mathrm{K}} \boldsymbol{B}$, then $h^{-1}[\theta] \in \operatorname{Con}_{\mathrm{K}} \boldsymbol{A}$, because $\boldsymbol{A} / h^{-1}[\theta] \in \mathbb{I S}(\boldsymbol{B} / \theta)$. As usual, ker $h$ denotes the kernel $h^{-1}\left[\mathrm{id}_{B}\right]$ of $h$.

A K-representation is an embedding $f: \boldsymbol{A} \rightarrow \prod_{i \in I} \boldsymbol{A}_{i}$, where $I$ is a set and each $\boldsymbol{A}_{i} \in \mathrm{K}$ (whence $\boldsymbol{A} \in \mathrm{K}$ ). We routinely identify $\boldsymbol{A}$ with its image $f[\boldsymbol{A}]$ and denote the representation by $\boldsymbol{A} \leqslant \prod_{i \in I} \boldsymbol{A}_{i}$. As usual, the representation is said to be subdirect if $\pi_{i}[A]=A_{i}$ for each $i \in I$, and direct if $A=\prod_{i \in I} A_{i}$. It is finitely indexed if the set $I$ is finite.

Definition 4.1. For a given K-representation $\boldsymbol{A} \leqslant \prod_{i \in I} \boldsymbol{A}_{i}$, a product congruence of $\boldsymbol{A}$ is a relation of the form $\theta=\left.\left(\otimes_{i \in I} \theta_{i}\right)\right|_{\boldsymbol{A}}$, where each $\theta_{i}$ belongs to the corresponding $\operatorname{Con} \boldsymbol{A}_{i}$ and

$$
\bigotimes_{i \in I} \theta_{i}:=\left\{\langle a, b\rangle \in\left(\prod_{i \in I} A_{i}\right)^{2}:\langle a(i), b(i)\rangle \in \theta_{i} \text { for all } i \in I\right\} .
$$

Clearly, $\theta \in \operatorname{Con} \boldsymbol{A}$. If $\theta_{i} \in \operatorname{Con}_{\mathrm{K}} \boldsymbol{A}_{i}$ for all $i$, we call $\theta$ a product Kcongruence; it is indeed a K-congruence of $\boldsymbol{A}$, because $\boldsymbol{A} / \theta$ embeds into $\prod_{i \in I} \boldsymbol{A}_{i} / \theta_{i} \in \mathrm{K}$. If every $\theta \in \operatorname{Con}_{\mathrm{K}} \boldsymbol{A}$ is a product K-congruence, the representation is said to admit only product $\mathrm{K}$-congruences.

In analogy with varieties, this last demand is related to the distributivity of $\mathrm{K}$-congruences. Indeed, the following conditions on $\mathrm{K}$ are equivalent:

(i) $\mathrm{K}$ is relatively congruence distributive.

(ii) Every finitely indexed subdirect K-representation admits only product K-congruences.

(iii) Every subdirect K-representation of the form $\boldsymbol{A} \leqslant \boldsymbol{B} \times \boldsymbol{B}$ admits only product $\mathrm{K}$-congruences. 
That (i) implies (ii) is an almost effortless generalization to quasivarieties of an old result of Foster and Pixley [25]; cf. [33, Theorem 4.1]. (All congruences introduced in the proof are K-congruences, by the Second Isomorphism Theorem.) The argument for (iii) $\Rightarrow$ (i) is as in Kiss [36, Lemma 1.2]. (The comments immediately after Kiss' proof reveal why his $\psi$ is a Kcongruence on the subalgebra of $\boldsymbol{A} \times \boldsymbol{A}$ with universe $\beta \circ \gamma$, provided that $\alpha, \beta, \gamma \in \operatorname{Con}_{\mathrm{K}} \boldsymbol{A}$. That is the only nontrivial step in the adaptation to quasivarieties.)

It follows from the equivalence of (i) and (ii) that a quasivariety $\mathrm{K}$ is $\mathrm{RCD}$ and has the RCEP iff every finitely indexed K-representation (not assumed subdirect) admits only product K-congruences. The argument for necessity uses the relation $\mathbb{S P} \leq \mathbb{P}_{\mathbb{S}} \mathbb{S}$; sufficiency includes a consideration of K-representations $\boldsymbol{A} \leqslant \boldsymbol{B}$ with only one factor.

In the analysis of infinitely indexed K-representations, other well-behaved $\mathrm{K}$-congruences arise naturally, just as for varieties. The join semilattice of compact (i.e., finitely generated) K-congruences of an algebra $\boldsymbol{A}$ shall be denoted as $\boldsymbol{C} \boldsymbol{o} \boldsymbol{m} \boldsymbol{p}_{\mathrm{K}} \boldsymbol{A}$. Of course, this is a join subsemilattice of $\boldsymbol{C o} \boldsymbol{n}_{\mathrm{K}} \boldsymbol{A}$, including $\operatorname{id}_{A}$.

Definition 4.2. Given a K-representation $\boldsymbol{A} \leqslant \prod_{i \in I} \boldsymbol{A}_{i}$, a filter $F$ over $I$ and an ideal $J$ of the join semilattice $\prod_{i \in I} \boldsymbol{C o m} \boldsymbol{p}_{\mathrm{K}} \boldsymbol{A}_{i}$, we define binary relations $\kappa_{F}$ and $\tau_{J}$ on $A$ as follows:

$$
\begin{aligned}
& \langle a, b\rangle \in \kappa_{F} \text { iff }\{i \in I: a(i)=b(i)\} \in F ; \\
& \langle a, b\rangle \in \tau_{J} \text { iff there exists } \eta \in J \text { with }\langle a(i), b(i)\rangle \in \eta(i) \text { for all } i \in I .
\end{aligned}
$$

Here, filters and ideals are assumed non-empty. A K-congruence of $\boldsymbol{A}$ is said to be filtral [resp. ideal] if it has the form $\kappa_{F}$ [resp. $\tau_{J}$ ] for some $F$ [resp. $J]$ as above. If this is true of all K-congruences of $\boldsymbol{A}$, we say that the representation $\boldsymbol{A} \leqslant \prod_{i \in I} \boldsymbol{A}_{i}$ admits only filtral [resp. ideal] K-congruences.

Note that $\kappa_{F}$ and $\tau_{J}$ are always K-congruences of $\boldsymbol{A}$. In particular, we have $\boldsymbol{A} / \kappa_{F} \in \mathbb{I S P}_{\mathbb{R}}(\mathrm{K}) \subseteq \mathrm{K}$, while $\boldsymbol{A} / \tau_{J}$ belongs to $\mathrm{K}$, as it satisfies any quasi-equation valid in $\mathrm{K}$ (use the fact that ideals are closed under finite joins).

Below, Lemma 4.3 and Theorem 4.5(iv),(v) generalize useful observations of Kiss, made without proof in Propositions 1.1 and 2.1 of [36], respectively. His claims were confined to subdirect representations (and absolute congruences), but need not have been. The meaning of 'ideal K-congruence' is clarified by Lemma 4.4. Theorem 4.5 confirms that ideal K-congruences are the natural generalization of product $\mathrm{K}$-congruences in the passage from finite to infinite products.

Lemma 4.3. Let $\boldsymbol{A} \leqslant \prod_{i \in I} \boldsymbol{A}_{i}$ be a K-representation, and let $X \subseteq A^{2}$. Then $\left.\Theta_{\mathrm{K}}^{\boldsymbol{A}} X \subseteq\left(\otimes_{i \in I} \Theta_{\mathrm{K}}^{\boldsymbol{A}_{i}} \pi_{i}[X]\right)\right|_{\boldsymbol{A}}$, where $\pi_{i}[X]:=\{\langle a(i), b(i)\rangle:\langle a, b\rangle \in X\}$. If $\Theta_{\mathrm{K}}^{\boldsymbol{A}} X$ is a product $\mathrm{K}$-congruence, then $\Theta_{\mathrm{K}}^{\boldsymbol{A}} X=\left.\left(\otimes_{i \in I} \Theta_{\mathrm{K}}^{\boldsymbol{A}_{i}} \pi_{i}[X]\right)\right|_{\boldsymbol{A}}$. 
Proof. The first assertion is obvious. Now suppose $\Theta_{\mathrm{K}}^{\boldsymbol{A}} X=\left.\left(\otimes_{i \in I} \theta_{i}\right)\right|_{\boldsymbol{A}}$, where $\theta_{i} \in \operatorname{Con}_{\mathrm{K}} \boldsymbol{A}_{i}$ for all $i$. Then, for each $i$, we have $\pi_{i}[X] \subseteq \theta_{i}$, whence $\Theta_{\mathrm{K}}^{\boldsymbol{A}_{i}} \pi_{i}[X] \subseteq \theta_{i}$. Therefore, $\left.\left.\left(\otimes_{i \in I} \Theta_{\mathrm{K}}^{\boldsymbol{A}_{i}} \pi_{i}[X]\right)\right|_{\boldsymbol{A}} \subseteq\left(\otimes_{i \in I} \theta_{i}\right)\right|_{\boldsymbol{A}}=\Theta_{\mathrm{K}}^{\boldsymbol{A}} X$.

Lemma 4.4. Let $\boldsymbol{A} \leqslant \prod_{i \in I} \boldsymbol{A}_{i}$ be a $\mathrm{K}$-representation, and $\theta$ a $\mathrm{K}$-congruence of $\boldsymbol{A}$. Then the following conditions are equivalent:

(i) $\theta$ is ideal;

(ii) for every finite subset $X$ of $\theta$, we have $\left.\left(\otimes_{i \in I} \Theta_{\mathrm{K}}^{\boldsymbol{A}_{i}} \pi_{i}[X]\right)\right|_{\boldsymbol{A}} \subseteq \theta$.

Proof. Let $\boldsymbol{P}$ denote the join semilattice $\prod_{i \in I} \boldsymbol{C o m p}_{\mathrm{K}} \boldsymbol{A}_{i}$. For each finite $X \subseteq \theta$, we define $\eta^{X} \in P$ by $\eta^{X}(i)=\Theta_{\mathrm{K}}^{\boldsymbol{A}_{i}} \pi_{i}[X], i \in I$.

(i) $\Rightarrow$ (ii): Suppose $\theta=\tau_{J}$, and consider $X=\left\{\left\langle a_{1}, b_{1}\right\rangle, \ldots,\left\langle a_{n}, b_{n}\right\rangle\right\} \subseteq \theta$. For each $k \in\{1, \ldots, n\}$, there exists $\mu_{k} \in J$ such that $\left\langle a_{k}, b_{k}\right\rangle \in \bigotimes_{i \in I} \mu_{k}(i)$. Let $\mu$ be the join, in $\boldsymbol{P}$, of $\mu_{1}, \ldots, \mu_{n}$. Then $\mu \in J$ and $\eta^{X} \leq \mu$. Thus, $\eta^{X} \in J$, and so $\left.\left(\otimes_{i \in I} \Theta_{\mathrm{K}}^{\boldsymbol{A}_{i}} \pi_{i}[X]\right)\right|_{\boldsymbol{A}} \subseteq \tau_{J}=\theta$.

(ii) $\Rightarrow$ (i): Let $J_{\theta}:=\left\{\eta \in P: \eta \leq \eta^{X}\right.$ for some finite $\left.X \subseteq \theta\right\}$. It is easy to see that $J_{\theta}$ is an ideal of $\boldsymbol{P}$, and that $\theta \subseteq \tau_{J_{\theta}}$, while (ii) supplies the reverse inclusion.

The claim in Lemma 4.4 could be paraphrased as: $\theta$ is ideal iff $\tau_{J_{\theta}} \subseteq \theta$. Also note that if $\theta$ is ideal, then $J_{\theta}$ is the smallest ideal $J$ of $\boldsymbol{P}$ for which $\theta=\tau_{J}$

Theorem 4.5. Consider a K-representation $\boldsymbol{A} \leqslant \prod_{i \in I} \boldsymbol{A}_{i}$.

(i) Every product K-congruence of $\boldsymbol{A}$ is ideal.

(ii) Every filtral K-congruence of $\boldsymbol{A}$ is ideal.

(iii) Every compact ideal $\mathrm{K}$-congruence of $\boldsymbol{A}$ is a product $\mathrm{K}$-congruence of the form $\left.\left(\bigotimes_{i \in I} \theta_{i}\right)\right|_{\boldsymbol{A}}$, with $\theta_{i} \in \operatorname{Comp}_{\mathrm{K}} \boldsymbol{A}_{i}$ for every $i \in I$.

(iv) The representation admits only ideal $\mathrm{K}$-congruences iff every compact $\mathrm{K}$-congruence of $\boldsymbol{A}$ is a product $\mathrm{K}$-congruence.

(v) When $I$ is finite, the ideal and product $\mathrm{K}$-congruences of $\boldsymbol{A}$ coincide.

Proof. (i) Let $\theta=\left.\left(\otimes_{i \in I} \theta_{i}\right)\right|_{\boldsymbol{A}}$, where $\theta_{i} \in \operatorname{Con}_{\mathrm{K}} \boldsymbol{A}_{i}$ for each $i \in I$. If $X \subseteq \theta$, then $\left.\left.\left(\otimes_{i \in I} \Theta_{\mathrm{K}}^{\boldsymbol{A}_{i}} \pi_{i}[X]\right)\right|_{\boldsymbol{A}} \subseteq\left(\bigotimes_{i \in I} \theta_{i}\right)\right|_{\boldsymbol{A}}=\theta$, so $\theta$ is ideal, by Lemma 4.4.

(ii) Let $F$ be a filter over $I$. For $a, b \in A$, let $W_{a b}=\{i \in I: a(i)=b(i)\}$. Let $X$ be a finite subset of $\kappa_{F}$. Then $W:=\bigcap_{\langle c, d\rangle \in X} W_{c d} \in F$. If $i \in W$, then $\pi_{i}[X] \subseteq \operatorname{id}_{A_{i}}=\Theta_{\mathrm{K}}^{\boldsymbol{A}_{i}} \pi_{i}[X]$, so if $\left.\langle a, b\rangle \in\left(\otimes_{i \in I} \Theta_{\mathrm{K}}^{\boldsymbol{A}_{i}} \pi_{i}[X]\right)\right|_{\boldsymbol{A}}$, then $W \subseteq$ $W_{a b}$, whence $W_{a b} \in F$, i.e., $\langle a, b\rangle \in \kappa_{F}$. Thus, $\left.\left(\otimes_{i \in I} \Theta_{K^{A_{i}}} \pi_{i}[X]\right)\right|_{\boldsymbol{A}} \subseteq \kappa_{F}$, and so $\kappa_{F}$ is ideal, by Lemma 4.4.

(iii) Let $\Theta_{\mathrm{K}}^{\boldsymbol{A}} X$ be an ideal K-congruence of $\boldsymbol{A}$, where $X \subseteq A^{2}$ is finite. Then $\Theta_{\mathrm{K}}^{\boldsymbol{A}} X=\left.\left(\otimes_{i \in I} \Theta_{\mathrm{K}}^{\boldsymbol{A}_{i}} \pi_{i}[X]\right)\right|_{\boldsymbol{A}}$, because Lemma 4.3 produces $\subseteq$ and Lemma 4.4 produces $\supseteq$. 
(iv) Necessity follows from (iii). Conversely, suppose $\theta \in \operatorname{Con}_{\mathrm{K}} \boldsymbol{A}$ and let $X \subseteq \theta$ be finite. Because $\Theta_{\mathrm{K}}^{\boldsymbol{A}} X$ is a product $\mathrm{K}$-congruence, Lemma 4.3 yields $\left.\left(\otimes_{i \in I} \Theta_{\mathrm{K}}^{\boldsymbol{A}_{i}} \pi_{i}[X]\right)\right|_{\boldsymbol{A}}=\Theta_{\mathrm{K}}^{\boldsymbol{A}} X \subseteq \theta$. Then $\theta$ is ideal, by Lemma 4.4.

(v) Let $\theta$ be an ideal K-congruence of $\boldsymbol{A}$. Then $\left.\theta \subseteq\left(\otimes_{i \in I} \Theta_{\mathrm{K}}^{\boldsymbol{A}_{i}} \pi_{i}[\theta]\right)\right|_{\boldsymbol{A}}$, by Lemma 4.3. We prove the reverse inclusion, which will establish (v) (in view of (i)). Let $\left.\langle a, b\rangle \in\left(\otimes_{i \in I} \Theta_{\mathrm{K}}^{\boldsymbol{A}_{i}} \pi_{i}[\theta]\right)\right|_{\boldsymbol{A}}$. For each $i \in I$, as $\Theta_{\mathrm{K}}^{\boldsymbol{A}_{i}}(a(i), b(i))$ is compact in $\boldsymbol{C o n}_{\mathrm{K}} \boldsymbol{A}_{i}$, we have $\langle a(i), b(i)\rangle \in \Theta_{\mathrm{K}}^{\boldsymbol{A}_{i}} \pi_{i}\left[X_{i}\right]$ for some finite $X_{i} \subseteq \theta$. Then $X:=\bigcup_{i \in I} X_{i}$ is a finite subset of $\theta$, because $I$ is finite. Now $\left.\langle a, b\rangle \in\left(\otimes_{i \in I} \Theta_{\mathrm{K}}^{\boldsymbol{A}_{i}} \pi_{i}[X]\right)\right|_{\boldsymbol{A}} \subseteq \theta$, where the last inclusion follows from Lemma 4.4.

The next result was stated without proof in [18, Theorem Q.9.2]; it was established for varieties in [27].

Theorem 4.6. ([18]) The following conditions on $\mathrm{K}$ are equivalent.

(i) $\mathrm{K}$ has parameterized EDPRC.

(ii) Every compact $\mathrm{K}$-congruence on a direct product of members of $\mathrm{K}$ is a product $\mathrm{K}$-congruence.

(iii) Every principal $\mathrm{K}$-congruence on a direct product of members of $\mathrm{K}$ is a product $\mathrm{K}$-congruence.

If these conditions hold and $\mathrm{K}$ has the RCEP, then $\mathrm{K}$ has EDPRC.

Proof. (i) $\Rightarrow$ (ii) follows from the first assertion of Lemma 4.3, together with Theorem 3.12, because pp sentences persist in the formation of direct products.

(ii) $\Rightarrow$ (iii) is trivial.

(iii) $\Rightarrow$ (i): We claim that some $\varphi \in \Phi_{\mathrm{K}}(x, y, z, w)$ defines principal relative congruences in K. Suppose, on the contrary, that for each $\varphi \in \Phi_{\mathrm{K}}(x, y, z, w)$, there exist $\boldsymbol{A}_{\varphi} \in \mathrm{K}$ and $a_{\varphi}, b_{\varphi}, c_{\varphi}, d_{\varphi} \in A_{\varphi}$ such that $\left\langle c_{\varphi}, d_{\varphi}\right\rangle \in \Theta_{\mathrm{K}}^{\boldsymbol{A}_{\varphi}}\left(a_{\varphi}, b_{\varphi}\right)$ but $\boldsymbol{A}_{\varphi} \not \models \varphi\left(a_{\varphi}, b_{\varphi}, c_{\varphi}, d_{\varphi}\right)$. Then $\boldsymbol{A}:=\prod_{\varphi \in \Phi_{\mathrm{K}}} \boldsymbol{A}_{\varphi} \in \mathrm{K}$. Let $a, b, c, d \in A$ be the elements whose respective $\varphi$-th co-ordinates are $a_{\varphi}, b_{\varphi}, c_{\varphi}, d_{\varphi}$. By (iii), $\Theta_{\mathrm{K}}^{\boldsymbol{A}}(a, b)$ is a product K-congruence, so it coincides with $\otimes_{\varphi \in \Phi_{\mathrm{K}}} \Theta_{\mathrm{K}}^{\boldsymbol{A}_{\varphi}}\left(a_{\varphi}, b_{\varphi}\right)$, by Lemma 4.3. Therefore, $\langle c, d\rangle \in \Theta_{\mathrm{K}}^{\boldsymbol{A}}(a, b)$. On the other hand, there is no $\varphi \in \Phi_{\mathrm{K}}$ for which $\boldsymbol{A}=\varphi(a, b, c, d)$, because pp sentences persist in homomorphic images. This contradicts Lemma 3.4, so $\mathrm{K}$ has parameterized EDPRC.

The last assertion follows from Corollary 3.6 and Theorem 3.9.

\section{Relatively ideal QUASIVARIETIES}

Definition 5.1. The quasivariety $\mathrm{K}$ is said to be relatively ideal if every representation of an algebra as a subdirect product of members of $\mathrm{K}_{R S I}$ admits only ideal K-congruences. 
'Ideal classes' (and 'filtral classes') were introduced around 1970 by R. Magari in a series of papers, including [41, 42, 43] (also see [3, 26]), but a relatively ideal quasivariety need not be a class of this kind, owing to the distinction between relative and absolute congruences. Definition 5.1 is phrased so as to minimize the burden of verification, but we can dispense quickly with the demand that the subdirect factors be K-subdirectly irreducible:

Lemma 5.2. Let $\mathrm{K}$ be a relatively ideal quasivariety. Then every subdirect $\mathrm{K}$-representation admits only ideal $\mathrm{K}$-congruences (even if the factors in the representation are not $\mathrm{K}$-subdirectly irreducible).

Proof. Let $\boldsymbol{A} \leqslant \prod_{i \in I} \boldsymbol{A}_{i}$ be a subdirect K-representation, with $\theta \in C o m p_{\mathrm{K}} \boldsymbol{A}$. For each $i \in I$, there exists a subdirect K-representation $\boldsymbol{A}_{i} \leqslant \prod_{j \in J_{i}} \boldsymbol{B}_{i j}$, with every $\boldsymbol{B}_{i j} \in \mathrm{K}_{R S I}$ (by Theorem 2.2), since $\boldsymbol{A}_{i} \in \mathrm{K}$. So, there is a subdirect K-representation $\boldsymbol{A} \leqslant \prod_{\langle i, j\rangle \in W} \boldsymbol{B}_{i j}$, where $W=\bigcup_{i \in I}\left(\{i\} \times J_{i}\right)$. Because $\mathrm{K}$ is relatively ideal, $\theta=\left.\left(\otimes_{\langle i, j\rangle \in W} \theta_{i j}\right)\right|_{\boldsymbol{A}}$ for suitable $\theta_{i j} \in C o m p_{\mathrm{K}} \boldsymbol{B}_{i j}$, $\langle i, j\rangle \in W$, by Theorem 4.5(iii). For each $i \in I$, let $\nu_{i}=\left.\left(\otimes_{j \in J_{i}} \theta_{i j}\right)\right|_{\boldsymbol{A}_{i}}$, so $\nu_{i} \in \operatorname{Con}_{\mathrm{K}} \boldsymbol{A}_{i}$. Then $\left.\left(\bigotimes_{i \in I} \nu_{i}\right)\right|_{\boldsymbol{A}}=\left.\left(\bigotimes_{\langle i, j\rangle \in W} \theta_{i j}\right)\right|_{\boldsymbol{A}}=\theta$, and Theorem 4.5(iv) delivers the result.

It also turns out that, in Lemma 5.2, we need not insist that the representation be subdirect, but that is less obvious. For varieties, it was essentially shown by Magari [42], but we shall use a simpler argument beginning (rather than ending) with a proof of the RCEP.

Theorem 5.3. Every relatively ideal quasivariety has the RCEP.

Proof. Let K be a relatively ideal quasivariety, and $\boldsymbol{A}$ a subalgebra of $\boldsymbol{B} \in \mathrm{K}$, with $a, b \in A$. It suffices to show that $\left.\Theta_{\mathrm{K}}^{B}(a, b)\right|_{\boldsymbol{A}} \subseteq \Theta_{\mathrm{K}}^{\boldsymbol{A}}(a, b)$.

For each $e \in B$, a function $f: \omega \rightarrow B$ is said to be almost constantly $e$ if $\{i \in \omega: f(i) \neq e\}$ is finite. Let $C$ be the set of all functions $f: \omega \rightarrow B$ such that $f$ is almost constantly $e$ for some $e \in A$. For any such $f$, there is just one such $e$, so we may write $e=h(f)$. Now $C$ is the universe of a subalgebra $\boldsymbol{C}$ of $\boldsymbol{B}^{\omega}$, and $h$ is a surjective homomorphism from $\boldsymbol{C}$ to $\boldsymbol{A}$. Clearly, $\pi_{i}[C]=B$ for each of the projections $\pi_{i}: B^{\omega} \rightarrow B, i \in \omega$.

For each $e \in B$, let $\bar{e}: \omega \rightarrow B$ be the constant function with range $\{e\}$. So, when $e \in A$, we have $\bar{e} \in C$ and $h(\bar{e})=e$. As the K-representation $\boldsymbol{C} \leqslant \boldsymbol{B}^{\omega}$ is subdirect, it admits only ideal K-congruences, by Lemma 5.2 , so $\Theta_{\mathrm{K}}^{C}(\bar{a}, \bar{b})$ is a product $\mathrm{K}$-congruence, by Theorem 4.5(iii). Thus, by Lemma 4.3,

$$
\Theta_{\mathrm{K}}^{C}(\bar{a}, \bar{b})=\left.\left(\bigotimes_{i \in \omega} \Theta_{\mathrm{K}}^{B}(a, b)\right)\right|_{C} .
$$

Now let $\left.\langle c, d\rangle \in \Theta_{\mathrm{K}}^{B}(a, b)\right|_{\boldsymbol{A}}$. Then $\left.\langle\bar{c}, \bar{d}\rangle \in\left(\bigotimes_{i \in \omega} \Theta_{\mathrm{K}}^{B}(a, b)\right)\right|_{\boldsymbol{C}}=\Theta_{\mathrm{K}}^{C}(\bar{a}, \bar{b})$. So, by Corollary 3.2, $\langle c, d\rangle=\langle h(\bar{c}), h(\bar{d})\rangle \in \Theta_{\mathrm{K}}^{\boldsymbol{A}}(h(\bar{a}), h(\bar{b}))=\Theta_{\mathrm{K}}^{\boldsymbol{A}}(a, b)$. We have shown that $\left.\Theta_{\mathrm{K}}^{\boldsymbol{B}}(a, b)\right|_{\boldsymbol{A}} \subseteq \Theta_{\mathrm{K}}^{\boldsymbol{A}}(a, b)$, as required. 
The above construction of $\boldsymbol{C}, h$ from $\boldsymbol{A}, \boldsymbol{B}$ has found other uses in the literature on ideal/filtral classes, e.g., [26, 36, 41, 42]. (Its first appearance was probably in [38], where it supplied a proof of the class operator relation $\mathbb{S} \leq \mathbb{H} \mathbb{P}_{\mathbb{S}}$ simpler than the ones in [16, p. 171] and [37].)

The next result has an analogue for varieties in [27, Theorems 4.5, 5.4], but the account there appears to contain a gap. (At the end of the proof of necessity, the CEP was tacitly relied on, but it was not established for ideal varieties in [27]. A longer indirect proof that ideal varieties have EDPC can be got by combining [36, Theorem 2.3], [39, Theorem 8] and Lemma 5.2.)

Theorem 5.4. A quasivariety is relatively ideal iff it has EDPRC.

Proof. $(\Rightarrow)$ Suppose the quasivariety $\mathrm{K}$ is relatively ideal. Then every principal K-congruence on a direct product of members of $\mathrm{K}$ is a product $\mathrm{K}$ congruence, by Lemma 4.5(iii). Also, $\mathrm{K}$ has the RCEP, by Theorem 5.3. Therefore, $\mathrm{K}$ has EDPRC, by Theorem 4.6.

$(\Leftarrow)$ Suppose $\mathrm{K}$ has EDPRC. Let $\boldsymbol{A} \leqslant \prod_{i \in I} \boldsymbol{A}_{i}$ be a K-representation, with $\theta=\Theta_{\mathrm{K}}^{\boldsymbol{A}} X$, where $X \subseteq A^{2}$ is finite. By Theorem 4.5(iv), it suffices to show that $\theta$ is a product $\mathrm{K}$-congruence. Let $\boldsymbol{B}=\prod_{i \in I} \boldsymbol{A}_{i}$. Then $\Theta_{\mathrm{K}}^{\boldsymbol{B}} X=$ $\otimes_{i \in I} \theta_{i}$ for suitable $\theta_{i} \in \operatorname{Con}_{\mathrm{K}} \boldsymbol{A}_{i}, i \in I$, by Theorem 4.6. But $\mathrm{K}$ has the RCEP, by Theorem 3.8, so $\theta=\left.\left(\Theta_{\mathrm{K}}^{B} X\right)\right|_{\boldsymbol{A}}=\left.\left(\otimes_{i \in I} \theta_{i}\right)\right|_{\boldsymbol{A}}$.

Note that the proof of Theorem 5.4 is also a proof of the following fact.

Corollary 5.5. Let $\mathrm{K}$ be a relatively ideal quasivariety. Then every $\mathrm{K}-r e p$ resentation admits only ideal $\mathrm{K}$-congruences (even if the representation is not subdirect and the factors not $\mathrm{K}$-subdirectly irreducible).

A relative subvariety of $\mathrm{K}$ is a class of the form $\mathrm{M}=\mathrm{K} \cap \mathrm{V}$, where $\mathrm{V}$ is a variety. (Equivalently, it is a subclass of $\mathrm{K}$ axiomatized, relative to $\mathrm{K}$, by some set of equations.) In this case, the $\mathrm{M}$-congruences and $\mathrm{K}$-congruences of algebras in $M$ coincide. So, properties like RCD, the RCEP, EDPRC and relative semisimplicity persist in relative subvarieties (not always in subquasivarieties).

Theorem 5.6. Let $\mathrm{M} \subseteq \mathrm{K}$ be quasivarieties, where $\mathrm{K}$ has EDPRC, and suppose $\mathrm{K} \cap \mathbb{H}\left(\mathrm{M}_{R S I}\right) \subseteq \mathrm{M}$. Then $\mathrm{M}$ is a relative subvariety of $\mathrm{K}$.

Proof. It suffices to prove that $\mathrm{K} \cap \mathbb{H}(\mathrm{M}) \subseteq \mathrm{M}$. Let $\boldsymbol{B} \in \mathrm{M}$ and $\theta \in \operatorname{Con}_{\mathrm{K}} \boldsymbol{B}$. By the Homomorphism Theorem, we need only show that $\boldsymbol{B} / \theta \in \mathrm{M}$. By Theorem 2.2, there is a subdirect K-representation $\boldsymbol{B} \leqslant \prod_{i \in I} \boldsymbol{B}_{i}$, with each $\boldsymbol{B}_{i} \in \mathrm{M}_{R S I} \subseteq \mathrm{K}$. By Theorem 5.4 and Lemma 5.2, $\theta$ is an ideal K-congruence of $\boldsymbol{B}$.

Let $\varphi$ abbreviate a $k$-variable quasi-equation $\left(\&_{j<n} \gamma_{j} \approx \delta_{j}\right) \Longrightarrow \gamma \approx \delta$ satisfied by M. It suffices to show that $\boldsymbol{B} / \theta=\varphi$.

Let $\boldsymbol{b}=\left\langle b_{1}, \ldots, b_{k}\right\rangle \in B^{k}$, where $X:=\left\{\left\langle\gamma_{j}^{\boldsymbol{B}}(\boldsymbol{b}), \delta_{j}^{\boldsymbol{B}}(\boldsymbol{b})\right\rangle: j<n\right\} \subseteq \theta$. Let $i \in I$, with $\theta_{i}=\Theta_{\mathrm{K}}^{\boldsymbol{B}_{i}} \pi_{i}[X]$. Then $\boldsymbol{B}_{i} / \theta_{i} \in \mathrm{K} \cap \mathbb{H}\left(\mathrm{M}_{R S I}\right)$, so $\boldsymbol{B}_{i} / \theta_{i} \in \mathrm{M}$, 
by assumption, whence $\boldsymbol{B}_{i} / \theta_{i} \models \varphi$. So, from

$$
\left\langle\gamma_{j}^{\boldsymbol{B}}(\boldsymbol{b})(i), \delta_{j}^{\boldsymbol{B}}(\boldsymbol{b})(i)\right\rangle \in \pi_{i}[X] \text { for all } j<n,
$$

we may infer $\left\langle\gamma^{\boldsymbol{B}}(\boldsymbol{b})(i), \delta^{\boldsymbol{B}}(\boldsymbol{b})(i)\right\rangle \in \theta_{i}$. Thus, $\left.\left\langle\gamma^{\boldsymbol{B}}(\boldsymbol{b}), \delta^{\boldsymbol{B}}(\boldsymbol{b})\right\rangle \in\left(\bigotimes_{i \in I} \theta_{i}\right)\right|_{\boldsymbol{B}}$, but $\left.\left(\bigotimes_{i \in I} \theta_{i}\right)\right|_{\boldsymbol{B}} \subseteq \theta$ (by Lemma 4.4), so $\boldsymbol{B} / \theta \models \varphi$.

Corollary 5.7. Suppose $\mathrm{M}$ is a subquasivariety of a variety with EDPC. If $\mathbb{H}\left(\mathrm{M}_{R S I}\right) \subseteq \mathrm{M}$, then $\mathrm{M}$ is a variety.

Remark 5.8. In Theorem 5.6 (and Corollary 5.7), if $M$ is known to be $\mathrm{RCD}$, then a sufficient condition for $\mathrm{K} \cap \mathbb{H}\left(\mathrm{M}_{R S I}\right) \subseteq \mathrm{M}$ is $\mathrm{K} \cap \mathbb{H}\left(\mathrm{M}_{F S I}\right) \subseteq \mathrm{M}$, because of Theorem 2.3(ii). Conversely, of course, any relative subvariety of $\mathrm{K}$ inherits EDPRC and is therefore RCD.

Blok and Pigozzi [10] showed that a quasivariety $\mathrm{K}$ has EDPRC iff the join semilattice $\boldsymbol{C o m p}_{\mathrm{K}} \boldsymbol{A}$ is dually Brouwerian for all $\boldsymbol{A} \in \mathrm{K}$. From this, they deduced the distributivity claim in Theorem 3.8. For varieties, these facts were proved earlier in [39] (and distributivity in [28] also). Kiss [36] gave a different proof that every ideal variety - i.e., every variety with EDPCis congruence distributive. His argument extends to quasivarieties as the combination of Theorem $4.5(\mathrm{v})$ and the implication (iii) $\Rightarrow$ (i) stated after Definition 4.1 (bearing Lemma 5.2 in mind).

A quasivariety has EDPRC iff it is RCD, with the RCEP, and has first order-definable principal relative congruences (DPRC); see [7, 10, 20]. DPRC follows even from parameterized EDPRC, by Corollary 3.10; it follows from the RCEP in locally finite quasivarieties (cf. [2]). The variety of semilattices has DPC [4] (and the CEP), but it lacks parameterized EDPC, because the square of the two-element semilattice violates condition (ii) of Theorem 4.6. Every subquasivariety of a directly representable quasivariety has DPRC [50]. In particular, every finitely generated semisimple congruence permutable variety has DPC, as it is directly representable [47, 48]. Eschewing local finiteness, the relatively semisimple RCD quasivariety CSR at the end of Section 2 lacks DPRC altogether. For $\boldsymbol{R} \in \mathrm{CSR}$, we have

$$
\langle c, d\rangle \in \Theta_{\mathrm{CSR}}^{\boldsymbol{R}}(a, b) \text { iff }(c-d)^{n}=(a-b) r \text { for some } r \in R \text { and some } n \in \mathbb{N} \text {. }
$$

The failure of DPRC is shown using a non-principal ultrapower $\boldsymbol{A}=\boldsymbol{R}^{\mathbb{N}} / U$ of the ring $\boldsymbol{R}$ of polynomials with rational coefficients in denumerably many commuting indeterminates $x_{1}, x_{2}, \ldots$ Setting $\bar{x}=\left\langle x_{1}, x_{2}, x_{3}, \ldots\right\rangle / U \in A$ and $\bar{y}=\left\langle x_{1}, x_{2}^{2}, x_{3}^{3}, \ldots\right\rangle / U \in A$, we find that $\left\langle x_{i}, 0^{\boldsymbol{R}}\right\rangle \in \Theta_{\mathrm{CSR}}^{\boldsymbol{R}}\left(x_{i}^{i}, 0^{\boldsymbol{R}}\right)$ for all $i \in \mathbb{N}$, but $\left\langle\bar{x}, 0^{\boldsymbol{A}}\right\rangle \notin \Theta_{\mathrm{CSR}}^{\boldsymbol{A}}\left(\bar{y}, 0^{\boldsymbol{A}}\right)$. Thus, by Łos' Theorem [13, Theorem V.2.9], CSR cannot have DPRC. It lacks the RCEP as well (consider $\mathbb{Z} \leqslant \mathbb{Q}$ ). It follows from an example of Quackenbush in [36, Theorem 3.9] that even a semisimple arithmetical variety with the CEP may lack DPC. (The example has denumerable type.) 


\section{Relatively FILTRAL QUASIVARIETIES}

Definition 6.1. The quasivariety $\mathrm{K}$ is said to be relatively filtral provided that every representation of an algebra as a subdirect product of members of $\mathrm{K}_{R S I}$ admits only filtral $\mathrm{K}$-congruences.

Lemma 6.2. Let $\boldsymbol{A} \leqslant \prod_{i \in I} \boldsymbol{A}_{i}$ be a K-representation, where each $\boldsymbol{A}_{i}$ is $\mathrm{K}$-simple. Then a $\mathrm{K}$-congruence of $\boldsymbol{A}$ is ideal iff it is filtral.

Proof. Filtral K-congruences are always ideal, by Theorem 4.5(ii). Conversely, let $J$ be an ideal of $\prod_{i \in I} \boldsymbol{C o m p}_{\mathrm{K}} \boldsymbol{A}_{i}$. As each $\boldsymbol{A}_{i}$ is K-simple, its total congruence is principal, so $\operatorname{Comp}_{\mathrm{K}} \boldsymbol{A}_{i}=\left\{\operatorname{id}_{A_{i}}, A_{i}^{2}\right\}$. For each $Y \subseteq I$, define $\theta^{Y} \in \prod_{i \in I} \operatorname{Comp}_{\mathrm{K}} \boldsymbol{A}_{i}$ so that $\theta^{Y}(i)=\operatorname{id}_{A_{i}}$ if $i \in Y$ and $\theta^{Y}(i)=A_{i}^{2}$ if $i \in I \backslash Y$. Then $F(J):=\left\{Y \subseteq I: \theta^{Y} \in J\right\}$ is a filter over $I$, because $\left|\operatorname{Comp}_{\mathrm{K}} \boldsymbol{A}_{i}\right|=2$ for all $i \in I$. And it is easy to see that $\kappa_{F(J)}=\tau_{J}$.

Theorem 6.3. A quasivariety is relatively filtral iff it is relatively semisimple and has EDPRC.

Proof. Given $\boldsymbol{A} \in \mathrm{K}_{R S I}$, where $\mathrm{K}$ is relatively filtral, consider the subdirect K-representation $\boldsymbol{A} \leqslant \boldsymbol{A}$ (with one factor). As there are just two filters over a singleton, and $\boldsymbol{A}$ is not trivial, $\boldsymbol{A}$ must be K-simple. Thus, every relatively filtral quasivariety is relatively semisimple. On the other hand, a relatively semisimple quasivariety is relatively filtral iff it is relatively ideal (by Lemma 6.2), iff it has EDPRC (by Theorem 5.4).

Filtral varieties (as opposed to Magari's 'filtral classes') were investigated by several authors in the early 1980s. The varietal specialization of Theorem 6.3 appeared in [27] and, with a different proof, in [28]. (In these papers, semisimplicity was assumed in the definition of filtrality.)

Corollary 6.4. Let $\mathrm{K}$ be a relatively filtral quasivariety. Then every $\mathrm{K}$-representation $\boldsymbol{A} \leqslant \prod_{i \in I} \boldsymbol{A}_{i}$, involving only $\mathrm{K}$-simple algebras $\boldsymbol{A}_{i}$, admits only filtral $\mathrm{K}$-congruences (even if the representation is not subdirect).

Proof. Use Theorems 6.3 and 5.4, Corollary 5.5 and Lemma 6.2.

For varieties, Corollary 6.4 follows from results of Magari [41].

By Theorem 6.3, the known characterizations of relatively semisimple quasivarieties with EDPRC all amount to relative filtrality. For a substantial list of such conditions, see Campercholi and Vaggione [14, Corollary 4.2] (in combination with [14, Theorem 4.1]). The next corollary records some of the desirable consequences of relative filtrality that follow from Theorem 6.3.

Corollary 6.5. In a relatively filtral quasivariety $\mathrm{K}$, the following hold.

(i) $\mathrm{K}$ is relatively congruence distributive, with the $R C E P$. 
(ii) For each $\boldsymbol{A} \in \mathrm{K}$, the set $\operatorname{Comp}_{\mathrm{K}} \boldsymbol{A}$ is closed under finite (non-empty) intersections, so it forms a sublattice of $\boldsymbol{C o n}_{\mathrm{K}} \boldsymbol{A}$. Moreover, this sublattice is itself a dual generalized Boolean lattice.

(iii) Every nontrivial algebra in $\mathrm{K}_{R F S I}$ is both $\mathrm{K}$-simple and finitely subdirectly irreducible in the absolute sense.

(iv) $\mathrm{K}_{R F S I}$ is a universal class.

Proof. Claim (i) follows from Theorems 3.8 and 6.3. When a quasivariety is $\mathrm{RCD}$, it is relatively semisimple with EDPRC iff it satisfies the first assertion of (ii) and that of (iii), by [14, Proposition 3.10, Corollaries 3.11, 4.2]. For the second assertion of (ii) (which goes back to [6] for varieties), see the remarks preceding [52, Lemma 5.3]. The second demand in (iii) follows from (i) and Theorem 2.3(ii), while (iv) follows from (i), (ii) and Theorem 2.3(iii).

The following theorem was proved by Czelakowski [17] under the assumption that $\mathrm{K}$ is a variety.

Theorem 6.6. Every relatively congruence distributive subquasivariety $\mathrm{M}$ of a relatively filtral quasivariety $\mathrm{K}$ is a relative subvariety of $\mathrm{K}$.

Proof. Because $\mathrm{M}$ is $\mathrm{RCD}$, it suffices to show that $\mathrm{K} \cap \mathbb{H}\left(\mathrm{M}_{F S I}\right) \subseteq \mathrm{M}$, in view of Theorems 6.3 and 5.6 and Remark 5.8. Now $\mathrm{M}_{F S I} \subseteq \mathrm{K}_{R F S I}$, which consists of K-simple algebras, by Corollary 6.5(iii), since $\mathrm{K}$ is relatively filtral. Thus, for any $\boldsymbol{B} \in \mathrm{M}_{F S I}$, the class $\mathrm{K} \cap \mathbb{H}(\boldsymbol{B})$ contains only trivial algebras and isomorphic copies of $\boldsymbol{B}$, whence it is contained in $\mathrm{M}$.

It was shown in $[5,28]$ that the congruence permutable filtral varieties are exactly the discriminator varieties. Some results about relatively semisimple quasivarieties with EDPRC in this spirit can be found in [14].

Theorems 6.3 and 2.1 allow us to infer Theorem 1.2 from Theorem 1.1, on purely algebraic grounds. These results square with known connections between deduction (-detachment) theorems and EDPRC [10, 18, 24], as a classical inconsistency lemma induces a deduction theorem in any algebraizable logic [52]. Of course, classical propositional logic and the variety of Boolean algebras witness Theorem 1.2. Another instance of Theorem 1.2 is revealed below, where we exhibit a relatively filtral quasivariety that is not a variety.

Example 6.7. Let $\boldsymbol{Z}_{3}=\left\langle Z_{3} ; \cdot, \rightarrow, \neg, \mathbf{t}, \perp\right\rangle$, where $Z_{3}=\{-1,0,1\}$ and $\cdot$ is the idempotent commutative monoid operation with identity $\mathbf{t}=0$, such that $-1 \cdot 1=-1=\perp$, and $\neg$ is the usual additive inversion on $Z_{3}$, while $a \rightarrow b=\neg(a \cdot \neg b)$ for all $a, b \in Z_{3}$. The tables for $\cdot$ and $\rightarrow$ in $Z_{3}$ are shown below.

\begin{tabular}{|c|c|c|c|c|c|c|}
\hline • & -1 & 0 & 1 & $\rightarrow$ & -1 & 0 \\
\hline-1 & -1 & -1 & -1 & -1 & 1 & 1 \\
\hline 0 & -1 & 0 & 1 & 0 & -1 & 0 \\
\hline 1 & -1 & 1 & 1 & 1 & -1 & -1 \\
\hline
\end{tabular}


It follows that for $a, b, c \in Z_{3}$, we have $c \leq a \rightarrow b$ iff $a \cdot c \leq b$, where the order is the standard one: $-1<0<1$.

The intensional fragments of the three-valued uninorm-based logic $\mathbf{I U M L} \mathbf{L}_{3}$ of $[46,49]$ (also see [1]) are algebraized by the quasivarieties $\mathrm{Q}=\mathbb{I S P}\left(\boldsymbol{Z}_{3}^{\mathfrak{S}}\right)$, where $\{\rightarrow\} \subseteq \mathfrak{S} \subseteq\{\cdot, \rightarrow, \neg, \mathbf{t}, \perp\}$ and $\boldsymbol{Z}_{3}^{\mathfrak{S}}$ is the $\mathfrak{S}$-reduct of $\boldsymbol{Z}_{3}$. The $\perp$-free reduct of $\boldsymbol{Z}_{3}$ differs from the better-known three-element Sugihara monoid only in that the latter has lattice operations $\wedge, \vee$ as well. The word 'intensional' signifies the exclusion of $\wedge, \vee$ from the signature, while $\rightarrow$ at least is retained.

Setting $|a|:=a \rightarrow a$, we see that the order on $Z_{3}$ is equationally definable in $\rightarrow$ alone: $a \leq b$ iff $a \rightarrow b=|a \rightarrow b|$ (iff $\mathbf{t} \leq a \rightarrow b$ ). Thus, the relation $\leq$ defined by $a \rightarrow b=|a \rightarrow b|$ partially orders all members of $\mathrm{Q}$. The anti-symmetry of $\leq$ is expressed by the quasi-equation

$$
(x \rightarrow y \approx|x \rightarrow y| \quad \& \quad y \rightarrow x \approx|y \rightarrow x|) \Longrightarrow x \approx y .
$$

We also define $a \leftrightarrow b=(a \rightarrow b) \cdot(b \rightarrow a)$, which is in fact the greatest lower bound of $a \rightarrow b$ and $b \rightarrow a$ in any member of Q; thus, $a=b$ iff $\mathbf{t} \leq a \leftrightarrow b$. The following quasi-equations (essentially from [1]) are satisfied by $\mathbf{Q}$.

$$
\begin{aligned}
& x \leq x \cdot y \Longleftrightarrow x \rightarrow|y| \leq x \rightarrow y \\
& \mathbf{t} \leq x \cdot y \Longrightarrow \mathbf{t} \leq x
\end{aligned}
$$

For all the intensional values of $\mathfrak{S}$, the quasivariety $Q$ is not a variety, as $\boldsymbol{Z}_{3}^{\mathfrak{S}}$ has a factor algebra, with universe $\{\{-1,1\},\{0\}\}$, that violates $(6)$ and is thereby excluded from $Q$.

The congruence yielding this factor algebra is the only proper non-identity congruence of $\boldsymbol{Z}_{3}^{\mathfrak{S}}$, so $\boldsymbol{Z}_{3}^{\mathfrak{S}}$ is subdirectly irreducible and Q-simple, but not simple. Any two-element algebra is simple, so $\mathbb{I S}\left(\boldsymbol{Z}_{3}^{\mathfrak{S}}\right)$ consists of Q-simple or trivial algebras. As $\boldsymbol{Z}_{3}^{\mathfrak{S}}$ is finite, we have $\mathrm{Q}_{R S I} \subseteq \mathbb{I S}\left(\boldsymbol{Z}_{3}^{\mathfrak{S}}\right)$, by Theorem 2.3(i), so $Q$ is relatively semisimple, whereas $\mathbb{H}(Q)$ is not semisimple.

Also, $Q$ has EDPRC. Indeed, it is implicit in [1] that, when $\mathfrak{S}$ includes • and $\rightarrow$, and $\boldsymbol{A} \in \mathrm{Q}$, then

$$
\langle c, d\rangle \in \Theta_{Q}^{\boldsymbol{A}}(a, b) \text { iff } a \leftrightarrow b \leq(a \leftrightarrow b) \cdot(c \leftrightarrow b) .
$$

Even in the absence of $\cdot$, the right hand side remains expressible, by (7). (That $\left.\mathbb{I S P}\left(\boldsymbol{Z}_{3}^{\rightarrow,}\right\urcorner\right)$ is RCD was shown in [23]. In contrast, the variety $\mathbb{H}(\mathrm{Q})$ satisfies no nontrivial congruence lattice identity [12].)

When $\mathfrak{S}$ includes $\perp$ (as well as $\rightarrow$ ), the algebras in $Q$ are bounded below by $\perp$ and above by $T=\perp \rightarrow \perp$. In summary, using Theorem 6.3, we infer:

Theorem 6.8. For $\{\rightarrow\} \subseteq \mathfrak{S} \subseteq\{\cdot, \rightarrow, \neg, \mathbf{t}, \perp\}$, the quasivariety $\mathrm{Q}=$ $\operatorname{ISP}\left(\boldsymbol{Z}_{3}^{\mathfrak{S}}\right)$ is relatively filtral, and not a variety. If, moreover, $\perp \in \mathfrak{S}$, then the nontrivial members of $\mathrm{Q}$ lack trivial subalgebras.

Thus, Theorem 1.2 is witnessed by the intensional fragments of $\mathbf{I U M L}_{3}$ with $\perp$. In the classical inconsistency lemma for the $\rightarrow, \perp$ fragment of 
$\mathbf{I U M L}_{3}$, the set $\Psi_{n}$ mentioned in Section 1 is $\left\{x_{1} \rightarrow \ldots \rightarrow x_{n} \rightarrow \perp\right\}$, for each positive integer $n$, where $x \rightarrow y \rightarrow z$ abbreviates $x \rightarrow(y \rightarrow z)$.

Acknowledgment. The second author thanks Tommaso Moraschini for many helpful comments, and for assistance with Italian-to-English translation.

\section{REFERENCES}

[1] A. Avron, Multiplicative conjunction as an extensional conjunction, Logic J. of the IGPL 5 (1997), 181-208.

[2] J.T. Baldwin, J. Berman, The number of subdirectly irreducible algebras in a variety, Algebra Universalis 5 (1975), 379-389.

[3] G.M. Bergman, Sulle classi filtrali di algebre, Ann. Univ. Ferrara, Sez. VII 27 (1971), $35-42$.

[4] J. Berman, Algebraic properties of $k$-valued logics, Proceedings of the Tenth International Symposium on Multiple-Valued Logic, Evanston, Illinois, 1980, pp. 195-204.

[5] W.J. Blok, P. Köhler, D. Pigozzi, On the structure of varieties with equationally definable principal congruences II, Algebra Universalis 18 (1984), 334-379.

[6] W.J. Blok, D. Pigozzi, On the structure of varieties with equationally definable principal congruences I, Algebra Universalis 15 (1982), 195-227.

[7] W.J. Blok, D. Pigozzi, Local deduction theorems in algebraic logic, in H. Andréka, J.D. Monk, I. Nemeti (eds.), 'Algebraic Logic', Colloquia Mathematica Societatis János Bolyai 54, Budapest (Hungary), 1988, pp. 75-109.

[8] W.J. Blok, D. Pigozzi, 'Algebraizable Logics', Memoirs of the American Mathematical Society 396, Amer. Math. Soc., Providence, 1989.

[9] W.J. Blok, D. Pigozzi, On the congruence extension property, Algebra Universalis 38 (1997), 391-394.

[10] W.J. Blok, D. Pigozzi, Abstract algebraic logic and the deduction theorem, manuscript, 1997. [See http://orion.math.iastate.edu/dpigozzi/for updated version, 2001.]

[11] W.J. Blok, J.G. Raftery, Ideals in quasivarieties of algebras, in X. Caicedo, C.H. Montenegro (eds.), 'Models, Algebras and Proofs', Lecture Notes in Pure and Applied Mathematics, Vol. 203, Marcel Dekker, New York, 1999, pp. 167-186.

[12] W.J. Blok, J.G. Raftery, Fragments of R-mingle, Studia Logica 78 (2004), 59-106.

[13] S. Burris, H.P. Sankappanavar, 'A Course in Universal Algebra', Graduate Texts in Mathematics, Springer-Verlag, New York, 1981.

[14] M.A. Campercholi, D.J. Vaggione, Implicit definition of the quaternary discriminator, Algebra Universalis 68 (2012), 1-16.

[15] M. Ćirić, S. Bogdanović, Posets of C-congruences, Algebra Universalis 36 (1996), 423-424.

[16] P.M. Cohn, 'Universal algebra', Harper \& Row, New York, 1965.

[17] J. Czelakowski, Relatively congruence-distributive subquasivarieties of filtral varieties, Bull. Sect. Logic 19 (1990), 66-70.

[18] J. Czelakowski, 'Protoalgebraic Logics', Kluwer, Dordrecht, 2001.

[19] J. Czelakowski, W. Dziobiak, Congruence distributive quasivarieties whose finitely subdirectly irreducible members form a universal class, Algebra Universalis 27 (1990), 128-149.

[20] J. Czelakowski, W. Dziobiak, The parameterized local deduction theorem for quasivarieties of algebras and its application, Algebra Universalis 35 (1996), 377-419.

[21] A. Day, A note on the congruence extension property, Algebra Universalis 1 (1971), 234-235. 
[22] W. Dziobiak, Finitely generated congruence distributive quasivarieties of algebras, Fund. Math. 133 (1989), 45-57.

[23] W. Dziobiak, Quasivarieties of Sugihara semilattices with involution, Algebra and Logic 39 (2000), 26-36.

[24] J.M. Font, R. Jansana, D. Pigozzi, A survey of abstract algebraic logic, and Update, Studia Logica 74 (2003), 13-97, and 91 (2009), 125-130.

[25] A.L. Foster, A.F. Pixley, Semi-categorical algebras II, Math. Z. 85 (1964), 169-184.

[26] R. Franci, Filtral and ideal classes of universal algebras, Quaderni dell'Instituto di Matematica dell'Universita di Siena (1976).

[27] E. Fried, G. Grätzer, R. Quackenbush, Uniform congruence schemes, Algebra Universalis $\mathbf{1 0}$ (1980), 176-189.

[28] E. Fried, E.W. Kiss, Connection between congruence-lattices and polynomial properties, Algebra Universalis 17 (1983), 227-262.

[29] V.A. Gorbunov, A characterization of residually small quasivarieties, Soviet Math. Dokl. 29 (1984), 204-207.

[30] V.A. Gorbunov, 'Algebraic Theory of Quasivarieties', Consultants Bureau, New York, 1998.

[31] G. Grätzer, 'Universal algebra', 2nd ed., Springer-Verlag, New York, 1979.

[32] G. Grätzer, H. Lakser, A note on the implicational class generated by a class of structures, Canad. Math. Bull. 16 (1973), 603-605.

[33] B. Jónsson, Congruence distributive varieties, Math. Japonica 42 (1995), 353-401.

[34] K. Kearnes, Relatively congruence distributive subquasivarieties of a congruence modular variety, Bull. Austral. Math. Soc. 41 (1990), 87-96.

[35] K. Kearnes, R. McKenzie, Commutator theory for relatively modular quasivarieties, Trans. Amer. Math. Soc. 331 (1992), 465-502.

[36] E.W. Kiss, Complemented and skew congruences, Ann. Univ. Ferrara, Sez. VII 29 (1983), 111-127.

[37] S.R. Kogalovskiı̆, Structural characteristics of universal classes (Russian), Sibirsk. Mat. Ž. 4 (1963), 97-119.

[38] S.R. Kogalovskiı̌, On Birkhoff's Theorem (Russian), Uspehi Mat. Nauk. 20 (1965), 206-207.

[39] P. Köhler, D. Pigozzi, Varieties with equationally definable principal congruences, Algebra Universalis 11 (1980), 213-219.

[40] J. Kollár, Congruences and one-element subalgebras, Algebra Universalis 9 (1979), 266-267.

[41] R. Magari, Varietà a quozienti filtrali, Ann. Univ. Ferrara, Sez. VII 14 (1969), 5-20.

[42] R. Magari, Varietà a congruenze ideali (Congruenze Ideali II), Ann. Univ. Ferrara, Sez. VII 15 (1970), 113-129.

[43] R. Magari, The classification of idealizable varieties (Congruenze Ideali IV), J. Algebra 26 (1973), 152-165.

[44] A.I. Maltsev, On the general theory of algebraic systems (Russian), Mat. Sbornik (N.S.) 35 (1954), 3-20.

[45] A.I. Maltsev, Several remarks on quasivarieties of algebraic systems (Russian), Algebra i Logika 5 (1966), 3-9.

[46] E. Marchioni, G. Metcalfe, Interpolation properties for uninorm based logics, Proceedings of ISMVL 2010, IEEE Computer Society Press (2010), 205-210.

[47] R. McKenzie, Para-primal varieties: A study of finite axiomatizability and definable principal congruences in locally finite varieties, Algebra Universalis 8 (1978), 336-348.

[48] R. McKenzie, Narrowness implies uniformity, Algebra Universalis 15 (1982), 67-85.

[49] G. Metcalfe, F. Montagna, Substructural fuzzy logics, J. Symbolic Logic 72 (2007), 834-864.

[50] A.M. Nurakunov, Quasivarieties of algebras with definable principal congruences, Algebra and Logic 29 (1990), 26-34. 
[51] D. Pigozzi, Finite basis theorems for relatively congruence-distributive quasivarieties, Trans. Amer. Math. Soc. 310 (1988), 499-533.

[52] J.G. Raftery, Inconsistency lemmas in algebraic logic, Math. Logic Quarterly 59 (2013), 393-406.

[53] W. Taylor, Residually small varieties, Algebra Universalis 2 (1972), 33-53.

[54] H. Tominaga, Some remarks on radical ideals, Math. J. Okayama Univ. 3 (1954), 139-142.

[55] C.J. van Alten, 'An Algebraic Study of Residuated Ordered Monoids and Logics without Exchange and Contraction', PhD. Thesis, University of Natal, Durban, 1998.

E-mail address: camper@famaf.unc.edu.ar

Ciem - Facultad de Matemática, Astronomía y Física (Fa.M.A.F.), Universidad Nacional de Córdoba - Ciudad Universitaria, Córdoba 5000, Argentina

E-mail address: james.raftery@up.ac.za

Department of Mathematics and Applied Mathematics, University of Pretoria, Private Bag X20, Hatfield, Pretoria 0028, South Africa 